\title{
Giant Virchow-Robin Spaces Presenting as Pseudo-Tumour of the Brainstem
}

\section{Bajaj SK ${ }^{1 *}$, Schnippering $\mathrm{H}^{2}$ and Albert $\mathrm{F}^{2}$}

${ }^{1}$ Department of Radiology, Klinikum Osnabrueck, Germany

${ }^{2}$ Department of Neurosurgery, Klinikum Osnabrueck, Germany

*Corresponding author: Satish Bajaj, Consultant Radiologist, Department of Radiology, Klinikum Osnabrueck, Am Finkenhuegel 1, 49076, Osnabrueck, Germany, Email: Satish. bajaj@Klinikum-os.de

\section{Case Report \\ Volume 4 Issue 1}

Received Date: February 19, 2020

Published Date: March 18, 2020

DOI: $10.23880 /$ crij-16000169

\section{Abstract}

We report a rare case of giant Virchow Robin spaces in the mesencephalon in a middle aged male suffering from ataxia, incomplete occulo-motor paresis, neurogenic urinary incontinence and cognitive impairment. A well document case with its MRI findings and clinical relevance are discussed.

Keywords: Virchow Robin Spaces; Perivascular Spaces; Magnet Resonance Imaging; Ataxia; Tumour

Abbreviations: VRSs: Virchow-Robin Spaces; MRI: Magnetic Resonance Imaging; CT: Computed Tomography; CSF: Cerebrospinal Fluid; MRS: Magnetic Resonance Spectroscopy; NAA: N-Acetyl-Aspartate.

\section{Introduction}

Virchow-Robin spaces (VRSs) are perivascular spaces of the brain which are pial-lined interstitial fluid-filled areas that accompany the penetrating arteries and arterioles. They penetrate the cerebral substance for a variable depth and appear as cystic structures, round or tubular in form. VRSs are a complex entity and are commonly seen in MRI scans in all age groups. Giant forms of VRSs are a rare entity and may mimic cystic neoplasia and may lead to mass effect. We present a well-documented case of giant VRSs of the mesencephalon with its MRI features and the relevant clinical manifestations.

\section{Case Report}

A middle aged male presented to the emergency department of our hospital with symptoms of progressive ataxia and loss of memory of recent onset. He also had episodes of vertigo and urinary incontinence for a year. The clinical examination revealed incomplete paresis of the right third cranial nerve and left sided ataxia. The initial CT scan showed no evidence of acute stroke or intracranial haemorrhages. Cystic changes were observed around the right sided Thalamus and in the midbrain with secondary extensively dilated third and lateral ventricle systems suggestive of an obstructive hydrocephalus (Figure 1).

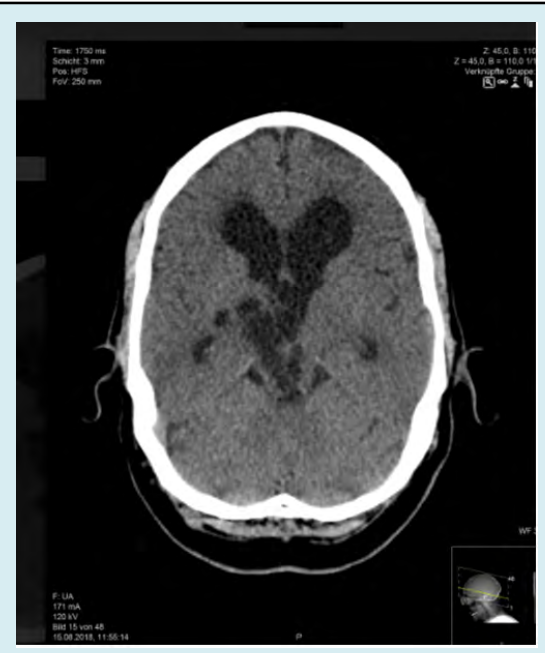

Figure 1: NECT showing cystic mass in the mesencephalon extending on the right sided thalamus with mass effect and secondary dilatation of the third and lateral ventricle. 
MRI scan of the brain showed bizarre, purely cystic mass of the mesencephalon extending to the right thalamus (Figures 2a-2d) with no significant oedema of the adjacent brain parenchyma. No diffusion restriction or accompanying haemorrhages within the lesion were seen. MRI also showed significant dilatation of the third and lateral ventricles. No enhancement was noted (Figures 3a \& 3b). The initial CT scan and MRI scan were suggestive of a probable tumour such as a Germinoma or an epidermoid cyst, or an Arteriovenous malformation. But the absence of diffusion restriction and contrast enhancement led to a probable diagnosis of unusual giant VRSs.

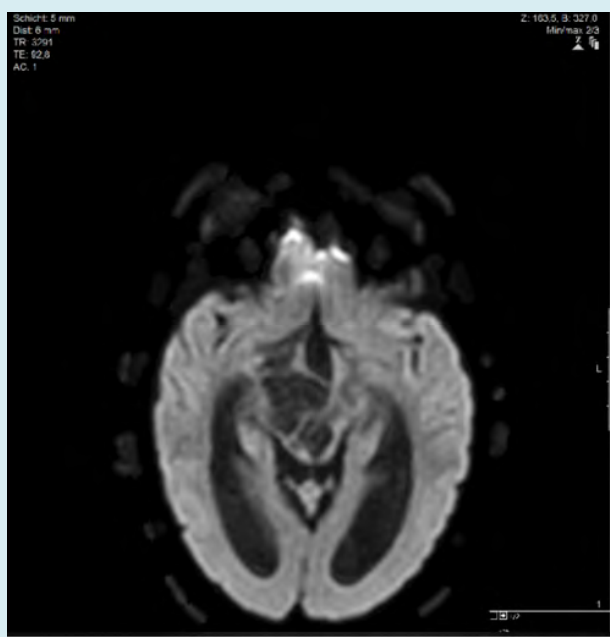

Figure 2a: Diffusion weighted (B1000) image axial view showing cystic lesions around thalamomegacephalon without any diffusion restrictions.

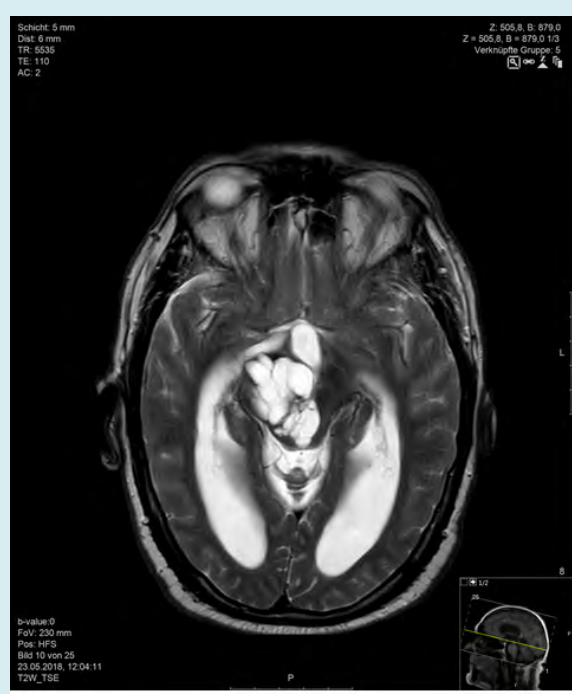

Figure 2b: Tw TSE axial view showing giant Virchow robin spaces as hyperintensies in the megacephalon with extension to thalamus on the right with secondary obstructive Hydrocephalus.

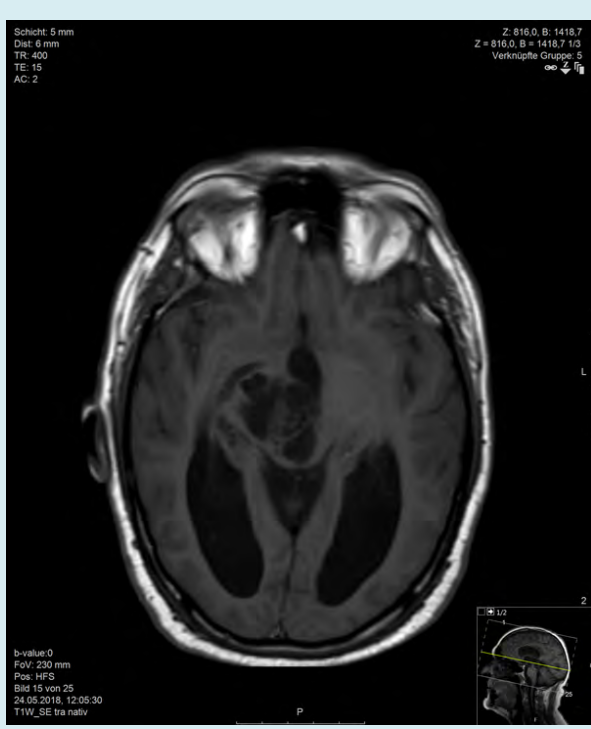

Figure 2c: T1w TSE axial view showing giant Virchow robin spaces as hypointensies in the megacephalon with extension to thalamus on the right with secondary obstructive Hydrocephalus.

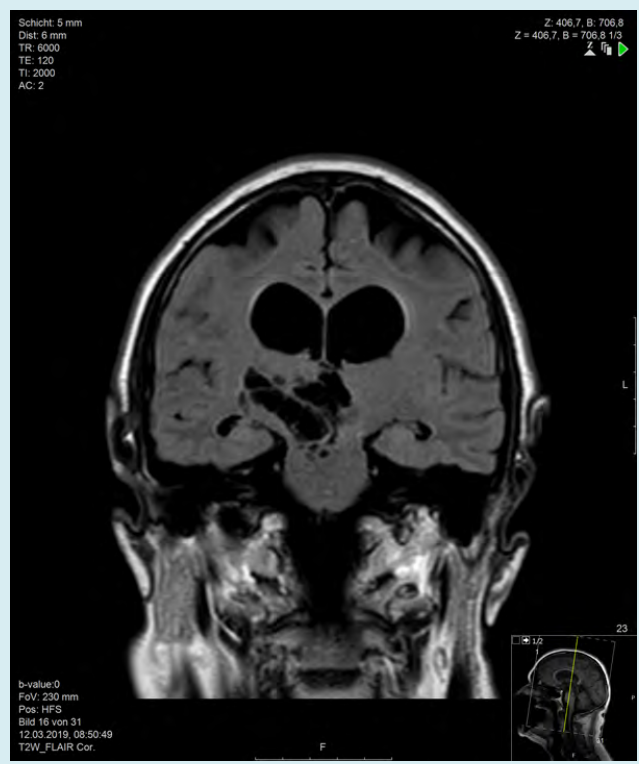

Figure 2d: Flair coronal view showing giant Virchow robin spaces as CSF isointense lesions in the mesencephalon with extension to thalamus on the right with secondary obstructive Hydrocephalus.

Results of Cerebrospinal fluid examinations, the EEG and the Duplex examination were within normal ranges. Funduscopic examinations of the eyes show no abnormal findings suggestive of raised intracranial pressure. The patient was treated conservatively without ventricle shunting/drainage which were reserved as an optional 


\section{Clinical Radiology \& Imaging Journal}

surgical management in case of persistence or deterioration of the symptoms. On follow-up after 3months on conservative medical treatment, the patient showed considerable improvement of symptoms. Follow-up CT and MRI scans showed no changes in the findings.

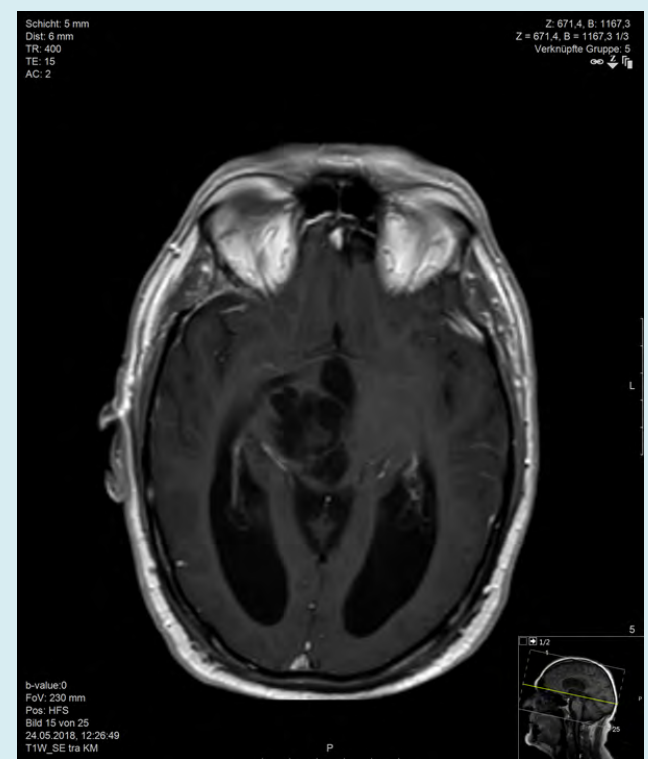

Figure 3a: T1w TSE axial with contrast showing giant Virchow robin spaces without any enhancement.

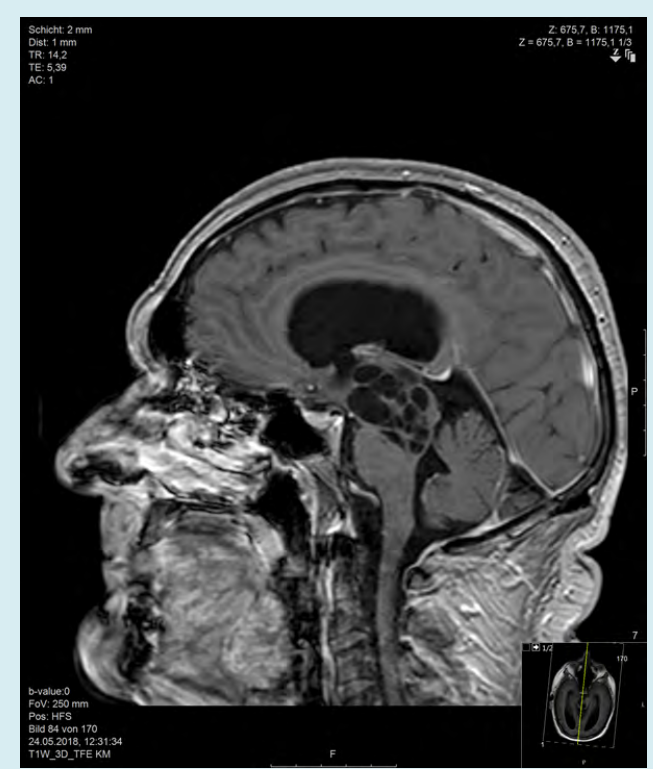

Figure 3b: T1w TSE sagittal with contrast showing giant Virchow robin spaces without any enhancement.

\section{Discussion}

Virchow-Robin spaces are named after Rudolf Virchow, a German pathologist and Charles Philippe Robin, a French anatomist [1,2]. Perivascular spaces or Virchow-Robin spaces are visualized as small CSF equivalent foci in all the sequences in MRI and follow the pathway of penetrating vessels from the subarachnoid space to various depths of penetration in the underlying brain substance. Small VRSs of 2-3mm size are a usual finding on routine MRI scans in any age group [3]. There is evidence suggesting that the VRSs may represent a lymphatic system draining the deep substance of the brain [4].

Several studies have shown that the increase in number of VRSs has a strong correlation with age, hypertension, dementia or other causes of brain atrophy [4,5]. It is also postulated that VRSs have a close association with neuropsychiatric disorders [5], multiple sclerosis [6] and mild traumatic brain injury [7]. Technically heavy weight T2 weighted sequence or MRI imaging with higher Tesla will delineate more VRSs. They should neither show diffusionrestrictions nor enhance on contrast sequences $[4,8]$. There are three types of VRSs as per their location $[6,9,10]$. Type 1 VRSs are seen along the course of lenticulostriate arteries located in basal ganglia whereas type 2 VRSs track towards the white matter from the convexity along the path of medullary arteries and veins and are located in both the hemispheres of the brain as well as in the cerebellum. The third types of VRSs are located along the course of the collicular and accessary collicular arteries in the ponto-mesencephalic junctions.

Giant Type I lesions should be differentiated from lacunar infarctions. These are unilateral compared to VRSs and show diffusion restriction in early stages as well as contrast enhancement [10]. Cystic periventricular leukomalacia in children and multiple sclerosis can mimic type II VRSs. Leukomalacia shows loss of white matter and ventricular dilatation whereas multiple sclerosis are oriented perpendicular to the corpus callosum which helps to differentiate them from VRSs [11]. Type II giant VRSs can mimic mucopolysaccharidosis which are inherited metabolic disorders of metabolism of glycosaminoglycans, leading to accumulation of toxic intracellular substrate [12]. In mucopolysaccharidosis, the brain changes are dominantly seen in white matter, corpus callosum and basal ganglia with increase in signal intensities in Flair and T2 weighted MRI sequences due to dysmyelination. In contrast, giant VRSs are signal hypointense in Flair sequences.

When VRSs become enlarged, they are known as giant VRSs or "cavernous dilatation". Giant VRSs type 3 are a rare presentation as in our case $[6,10]$. They are located in the mesencephalothalamic region in the territory of mesencepahlothalamo-thalamic (collicular) artery as mentioned above [6,13]. Obstruction of CSF drainage pathways could be a major factor in the pathogenesis of giant VRSs, although the exact nature of the obstruction is 


\section{Clinical Radiology \& Imaging Journal}

unclear. They may cause compression of the third ventricle or Aqueduct leading to hydrocephalus and may require surgical intervention $[13,14]$. Such giant form of VRSs may be associated with $\mathrm{T} 2 \mathrm{w}$ and Flair changes in the surrounding areas due to oedema with weird MRI findings prompting a tissue biopsy. To avoid unnecessary interventions, conditions with similar features and similar locations should be considered in the differential diagnosis.

Giant VRSs in the mesencephalon can mimic infectious pathologies, in particular Cryptococcosis, an opportunistic fungal infection caused by Cryptococcus neoformans in immunocompromised and HIV patients. The infection extends along the VRSs and causes cystic changes appearing as Pseudocysts, mainly in basal ganglia, thalami and midbrain. They may show diffusion restrictions due to high viscosity of the infective materials and hyperintense signals in T2 weighting and Flair images as well as contrast enhancement which differentiate them from VRSs [15]. Cystic neoplasia of the mesencephalon, for example cystic glioma, epidermoid and teratoma are not so uncommon. Solid components, accompanying oedema, contrast enhancement and diffusion restriction, in particular in case of epidermoid cysts differentiate them from true VRSs [16].

Arachnoid cysts and Neuroepithethial cysts should be considered in differential diagnosis, because they share similar MRI feature like CSF isointense cystic mass in all the MRI sequences. These congenital cystic pathologies differ in their form and location (peri-mesencephalic) from the giant VRSs in the thalamo-mesencaphalon [6]. MR-Spectroscopy (MRS) is a very good tool to exclude few conditions which are commonly seen in the midbrain [17]. In cases of cystic tumours, MRS will show a reduction in NAA and increase in choline along with lactate and lipids. Abscess and parasitic cysts show increase in lactate, succinate and amino acids in the absence of normal brain metabolites. MRS is usually normal in giant VRSs, except in few cases in which increase in lactate and choline can be seen due to secondary demyelination [18-20].

VRSs usually cause no mass effect or perilesional gliosis. They are seldom symptomatic due to hydrocephalus or increasing mass effect on the surrounding structure. Headache, dizziness, dementia, visual changes, cranial neuropathy, seizure, syncope, stroke, memory problem, poor balance and concentration are the common manifestations resulting from giant VRSs $[6,10]$. The prognosis and the course of giant VRSs are not predictable. Over a time they may show further increase in size with secondary deterioration of symptoms. Surgical interventions such as ventriculostomy, ventriculoperitoneal or cystoperitoneal shunt may be required due to persistent symptomatic hydrocephalus [2123]. In conclusion, VRSs should be considered in differential diagnosis in patients with large cystic lesions without enhancement located mainly in the mesencephalon. This can, as in our patient, help avoid unnecessary neurosurgical interventions.

\section{References}

1. Virchow $\mathrm{R}$ (1851) ueber die Erweiterung kleiner gefaesse. Archiv Pathol Anat Physiol klin Med 3: 427462.

2. Robin C (1859) Recherches sur quelues particularites de la structure des capillaires e l'encephale. J Physiol Homme Anim 2: 537-548.

3. Pollock H, Hutchings M, Weller RO, Zhang ET (1997) Perivascular spaces in the basal ganglia of the human brain: their relationship to lacunaes. J Anat 191(3): 337346.

4. Schley D, Carare-Nnadi R, Pleases CP, Perry VH, Weller RO (2006) Mechanism to explain the reverse perivascular transport of solutes out of brain. J Theor Biol 238(4): 962-974.

5. Poirier J, Gray F, Gherardi R, Derouesne C (1985) Cerebral lacunae: A new Neuropathological classification. J Neuropathol Exp Neurol 44: 312.

6. Kwee RM, Kwee TC (2007) Virchow robin spaces at MR imaging. Radiographics 27(4): 1071-1086.

7. Heier LA, Bauer CJ, Schwartz L, Zimmerman RD, Morgello S, et al. (1989) Large Virchow robin spaces: MR -clinical correlation. AJNR Am J Neuroradiol 10(5): 926-936.

8. Saeki N, Sato M, Kubota M, Uchino Y, Murai H, et al. (2005) MR imaging of normal perivascular space expansion at Midbrain. AJNR AM J Neuro-Radiol 26(3): 566-571.

9. Demaerel P, Wilms G, Baert AL, Van der Bergh V, Sainte $\mathrm{T}$ (1996) Widening of Virchow robin spaces. AJNR Am J Neuroradiol 17(4): 800-801.

10. Salzmann KL, Osborn AG, House P, Jinkins JR, Ditchfield A, et al. (2005) Giant tumefactive perivascular spaces. AJNR Am J neuro-radiol 26: 298-305.

11. Pretorius PM, Quaghebeur G (2003) The role of MRI in the diagnosis of MS. Clin Radiol 58(6): 434-448.

12. Poiier J, Barbizet, Gaston A, Meyrignac C (1983) Demence thalamique:lacunes expansives du territoire thalamo-mesencephalique paramedian. Hydrocephalus oar stenose denfaqueduc de sylvius. Rev Neurol (Paris) 139: 349-358. 
13. Kanamalla US, Calabro R, Jinkins JR (2000) Cavernous dilatation of mesencephalic Virchow-Robin spaces with obstructive hydrocephalus. Neuroradiology 42(12): 881-884.

14. Miszkiel KA, Hall-Craggs MA, Miller RF, Kendall BE, Wilkinson ID, et al. The spectrum of MRI findings in CNS cryptococcosis in AIDS. Clin Radiol 51(12): 842-850.

15. Papayannis CE, Saidon P, Rugilo A, Hess D, Rodriguez G, et al. (2003) Expanding Virchow Robin spaces in the midbrain causing Hydrocepahlus. AJNR Am J Neuroradiol 24(7): 1399-1403.

16. Mascalchi M, Salvi F, Godano U, Nistri M, Taiuti R, et al. (1999) Expanding lacunae causing triventricular hydrocephalus: report of two cases. J Neurosurg 91(4): 669-674.

17. Poptani H, Gupta RK, Jain VK, Roy R, Pandey R (1995) Cystic intracranial mass lesions: possible role of in vivo MR spectroscopy in its differential diagnosis. Magn Reson Imaging 13(7): 1019-1029.

18. MacLullich AM, Wardlaw JM, Ferguson KJ, Starr JM, Seckl JR, et al. (2004) Enlarged perivascular spaces are associated with cognitive function in healthy elderly men. J Neuro Neurosurg Psychiatry 75(11): 1519-1523.

19. Achiron A, Faibel M (2002) Sandlike appearance of Virchow robin spaces in early multiple sclerosis: a novel Neuroradiologic marker. AJNR Am J Neuro-radiol 23(3): 376-380.

20. Inglese $\mathrm{M}$, Bomsztyk E, Gonen O, Mannon LJ, Grossman RI, et al. (2005) Dilated perivascular spaces: hallmarks of mild traumatic brain injury. AJNR Am J Neuroradiol 26(4): 719-724.

21. Matheus MG, Castillo M, Smith JK, Armao D, Towle D, et al. (2004) Brain MRI findings in patients with mucopolysaccharidosis type I and II and mild clinical presentation. Neuroradiology 46(8): 666-672.

22. Tien RD, Flesberg GJ, Friedman H, Brown M, Macfall J (1994) MR imaging of high grade cerebral gliomas: value of diffusion weighted echoplanar pulse sequences. AJR Am J Roenthenol 162(3): 671-677.

23. Guermazi A, Miaux Y, Majoulet JF, Lafitte F, Cghiras J (1998) Imaging findings of central nervous system neuroepithetial cysts. Eur Radiol 8(4): 618-623. 